\title{
Characterization of mitochondria in cisplatin- resistant human ovarian carcinoma cells
}

\author{
MASANORI HIRAMA, SEIJI ISONISHI, MAKOTO YASUDA and HIROSHI ISHIKAWA \\ Department of Obstetrics/Gynecology and Department of Anatomy II, Jikei University \\ School of Medicine, 3-25-8 Nishi-shinbashi, Minato-ku, Tokyo 105, Japan
}

Received June 5, 2006; Accepted July 20, 2006

\begin{abstract}
One of the mechanisms of cisplatin cell cytotoxicity is the mitochondria-associated induction of apoptosis. The morphological or functional change of mitochondria in cisplatin-resistant cells has already been reported. Herein we present additional data describing the mitochondrial genomic and functional changes in cisplatinresistant cells. Cisplatin increased the level of apoptotic cells in cisplatin-sensitive human ovarian carcinoma OV 2008 and C13 cells by $3.90 \pm 1.01(\mathrm{SD} ; \mathrm{N}=3)(\mathrm{p}<0.01)$-fold and $2.03 \pm 0.20(\mathrm{SD} ; \mathrm{N}=3)(\mathrm{p}<0.01)$-fold compared to the basal apoptotic level. This indicates a lower level induction of apoptosis by $50 \%$ in cisplatin-resistant OV 2008/C13*5.25 variant (C13) cells. In both cell types, cisplatin cytotoxicity is mostly inhibited by the caspase- 9 inhibitor as well as the caspase-3 inhibitor, Ac-DEVD-CHO, suggesting that the mitochondrial downstream event was functioning well in both the C13 cells and in OV 2008 cells. Mitochondrial transmembrane potential $(\Delta \Psi \mathrm{m})$ determined by flow cytometry using $\mathrm{DiOC}_{6}$-stained cells revealed a significant depolarization of $\mathrm{C} 13$ cells as compared to OV 2008 cells. Treatment of these cells with cisplatin or hydrogen peroxide induces complete mitochondrial DNA damage in OV 2008 cells, while only partial DNA-destruction is observed in C13 cells, strongly suggesting that mitochondria are resistant to cisplatin and oxidative stress response. Continuous oxygen consumption of these cells monitored by a multi-channel dissolved oxygen meter is 1.70-fold higher in OV 2008 cells than $\mathrm{C} 13$ cells and the oxygen consumption was decreased by $30 \%$ in $\mathrm{C} 13$ cells, suggesting mitochondrial respiratory
\end{abstract}

Correspondence to: Dr Seiji Isonishi, Department of Obstetrics/ Gynecology, Jikei University School of Medicine, 3-25-8 Nishishinbashi, Minato-ku, Tokyo 105, Japan

E-mail: isonishi@jikei.ac.jp

Abbreviations: $\mathrm{IC}_{50}$, drug concentration causing $50 \%$ inhibition of colony formation

Key words: mitochondrial DNA, apoptosis, cisplatin resistance, ovarian carcinoma malfunction in these cells. The hypothesis generated here is that mitochondrial DNA resistance to cisplatin and oxidative stress response might be one of the main characteristics concerning the lower level of apoptosis induced by cisplatin. However, the mechanism by which the mitochondrial DNA encoded molecule is involved in cisplatin resistance remains to be determined.

\section{Introduction}

Platinum drugs remain one of the most important chemotherapeutic agents for ovarian cancer treatment. The resistance to cisplatin is a major obstacle for successful cancer therapy. Although significant biochemical changes in the cisplatinresistant cells including increased drug efflux, decreased drug influx, increased cellular glutathione level, and DNA repair function have been described, the mechanisms resulting in cellular cisplatin resistance remains unclear. A wide range of chemotherapeutic drugs including cisplatin induce death in malignant cells by triggering apoptosis (1). Cisplatin specifically has been shown to induce apoptosis by i) induction of the Fas ligand on the surface of tumor cells, resulting in killing of the Fas-sensitive subpopulation, ii) the direct activation of caspase-8, and iii) the integration of various cell stress signals via the 'activation' of mitochondria. One crucial event in the apoptotic pathway is the cytochrome $c$ release from the mitochondria into the cytosol. Cytochrome $c$ initiates the assembly of the apoptotic protease-activating factor 1 and procaspase-9 into a holoenzyme complex which in turn activates the initiator caspase-9. This activates downstream caspase-3, resulting in DNA fragmentation and apoptosis. A current view of drug-induced apoptosis emphasizes the role of different cell organelles as stress sensors that reroute the signal via mitochondria or other pathways $(2,3)$. Alterations in mitochondria $(4,5)$ or its associated apoptotic pathway play an important role in acquiring drug resistance. There are two distinct hypotheses concerning the correlation between drug sensitivity and mitochondria. Liang and Ullyatt (6) reported an increased cisplatin sensitivity in mitochondrial DNA depleted U937 cells. They demonstrated that these cells continued to respond to cisplatin by an apoptotic death. Thus mitochondrial DNA-encoded molecules may play a role in the sensitivity of cells to undergo a cisplatin-induced apoptotic death, but may not be required for cells to undergo apoptosis. 
On the other hand, Park et al (7) have shown that mitochondrial DNA-depleted SK-Hep1 hepatoma cells with frequent mitochondrial DNA mutations are resistant to various forms of cell death, such as oxidative stress and chemotherapeutic agents.

In the present study, we have focused on the characteristics of mitochondria in cisplatin resistant cells, particularly the direct interaction between cisplatin and mitochondrial DNA. We will show that platinum-resistant cells are characterized by mitochondrial respiratory abnormalities and contain mitochondrial DNA with resistance to cisplatin and to reactive oxygen. The hypothesis generated here is that the mitochondrial DNA encoded molecules might participate in the resistance to cisplatin-induced apoptosis.

\section{Materials and methods}

Reagents. Cisplatin was obtained from Bristol-Myers Squibb K.K. Hoechst-33342 was purchased from Sigma Chemical Co. $\mathrm{DiOC}_{6}$ was purchased from Molecular Probes, Inc. The caspase-3 inhibitor Ac-DEVD-CHO and caspase-9 inhibitor were purchased from Calbiochem, La Jolla, CA.

Tumor cell lines. The human cell line OV 2008 was established from a patient with a serous cystadenocarcinoma of the ovary (8). A resistant subline, designated OV 2008/C13*5.25 (C13), was obtained by 13 monthly selections to cisplatin exposure. The cells were cultured at $37^{\circ} \mathrm{C}$ in $95 \%$ air and $5 \% \mathrm{CO}_{2}$. They were grown in RPMI-1640 medium supplemented with $5 \%$ heat inactivated fetal bovine serum, $2 \mathrm{mM}$ glutamine, 100 Units/ml penicillin, and $100 \mu \mathrm{g} / \mathrm{ml}$ streptomycin.

Colony assays. Colony forming assays were used to assess the effect of the caspase-3 inhibitor Ac-DEVD-CHO and caspase-9 inhibitor on drug sensitivity. Cell suspensions, containing 600 cells, were plated on tissue culture dishes. Drug solution was added to triplicate plates at each drug concentration. After $30 \mathrm{~min}$ preincubation in the presence of caspase inhibitor, cells were treated with cisplatin and either one of the above inhibitors concurrently for $72 \mathrm{~h}$. Control cells were incubated with either cisplatin or the inhibitor or vehicle alone. Following drug exposure the drug-containing medium was aspirated and replaced with a drug free medium. Colonies of over 60 cells were counted macroscopically.

Detection of apoptosis. Nuclear staining by Hoechst-33342 was viewed and photographed using Zeiss fluorescence microscope. Cells with typical apoptotic nuclear morphology such as nuclear shrinkage, fragmentation, and condensation were identified and counted using randomly selected fields on numbered slides with the counter unaware of the treatment, so as to avoid experimental bias. The percentage of induction of apoptosis was estimated by counting these cells in duplicate samples of 400 cells.

Assessment of mitochondrial membrane potential ( $\Delta \Psi m)$. The cationic lipophilic fluorochrome $\mathrm{DiOC}_{6}$ (9) (Molecular Probes, Eugene, OR, USA) was used to measure the $\Delta \Psi \mathrm{m}$. The cells were incubated for $12 \mathrm{~min}$ at $37^{\circ} \mathrm{C}$ in the presence of the fluorochrome, washed in $\mathrm{PBS} / 1 \% \mathrm{FCS}$, and immediately analyzed by flow cytometry (FACScan). $\mathrm{DiOC}_{6}$ (9) was recorded in fluorescence. The percentage of cells with low mitochondrial potential was calculated in comparison with the untreated control cells.

Isolation of mitochondria. Mitochondria were isolated from OV 2008 and C13 cells by a modification of the method of Maltese and Aprille (10). Typically, $5 \times 10^{8}$ cells were harvested in DMEM. Cells were pelleted and washed once with homogenization buffer $(250 \mathrm{mM}$ sucrose, $1 \mathrm{mM}$ Tris- $\mathrm{HCl}$, and $1 \mathrm{mM}$ EDTA, and $1 \mathrm{mg} / \mathrm{ml} \mathrm{BSA}, \mathrm{pH} 7.4)$. The final pellet of cells was resuspended in homogenization buffer to a volume of $7 \mathrm{ml}$ and homogenized in a tissue grinder with a tight pestle until at least $95 \%$ of the cells were disrupted. The homogenate was centrifuged at $800 \mathrm{x}$ g for $10 \mathrm{~min}$ at $4^{\circ} \mathrm{C}$. The supernatant was removed and saved, and the pellet was resuspended and centrifuged again at $800 \mathrm{x}$ g for $10 \mathrm{~min}$. The supernatants were then pooled and centrifuged at 9,400 x g for $10 \mathrm{~min}$ at $4^{\circ} \mathrm{C}$. The pellet was resuspended in homogenization buffer and centrifuged again at 9,400 x g, and the final pellet was suspended in homogenization buffer to a final volume of about $5 \mathrm{mg}$ protein $/ \mathrm{ml}$.

Detection of mitochondrial DNA damage. DNA was extracted according to the instructions of the manufacturer. To detect mitochondrial DNA damage, the entire coding region of the mitochondrial genome was amplified by long range PCR with the use of primers 59-CCCACAGTTTATGTAGC TTACCTCCTCA-39 (nucleotides 571-598 of the Cambridge human mitochondrial DNA sequence) and 59-TTGATTGCT GTACTTGCTTGTAAGCATG-39 (nucleotides 16,22016,193). Long range PCR was performed with the Expand Long Template PCR system (Boehringer Mannheim) according to the manufacturer's instructions with the following modifications: Denaturing temperature $92^{\circ} \mathrm{C}$, annealing temperature $65^{\circ} \mathrm{C}$, extension time 14 min during the first 10 cycles and $14 \mathrm{~min}$ plus $20 \mathrm{sec}$ for each consecutive cycle during the subsequent 12 cycles. The final extension time was $14 \mathrm{~min}$. The 15.6-kb PCR products were resolved on a $0.7 \%$ agarose gel and visualized by ethidium bromide. As a positive control, mitochondrial DNA damage was induced in the OV 2008 or $\mathrm{C} 13$ cells by treatment with $\mathrm{H}_{2} \mathrm{O}_{2}$ in concentrations ranging from 6.25 to $50 \mu \mathrm{M}$ at $37^{\circ} \mathrm{C}$ for $1 \mathrm{~h}$. To detect cisplatin-induced DNA damage, OV 2008 and C13 cells, respectively, were treated with cisplatin.

Measurement of oxygen consumption. Cellular oxygen consumption was determined by disposable oxygen electrode sensor and multi-channel dissolved oxygen meter (Daikin Co. DOX-96). The continuous oxygen consumption (slop of dissolved oxygen, SDO) and the accumulated total amount of oxygen consumption for $8 \mathrm{~h}$ (difference in the area under the curve, AUC) were calculated in OV 2008 and C13 cells.

Statistical analysis. Differences between samples or the groups of samples were determined by Student's t-test using two-sided p-values.

\section{Results}

Apoptotic fraction. The percentage of induction of apoptosis was estimated by counting the frequency of Hoechst-33342 


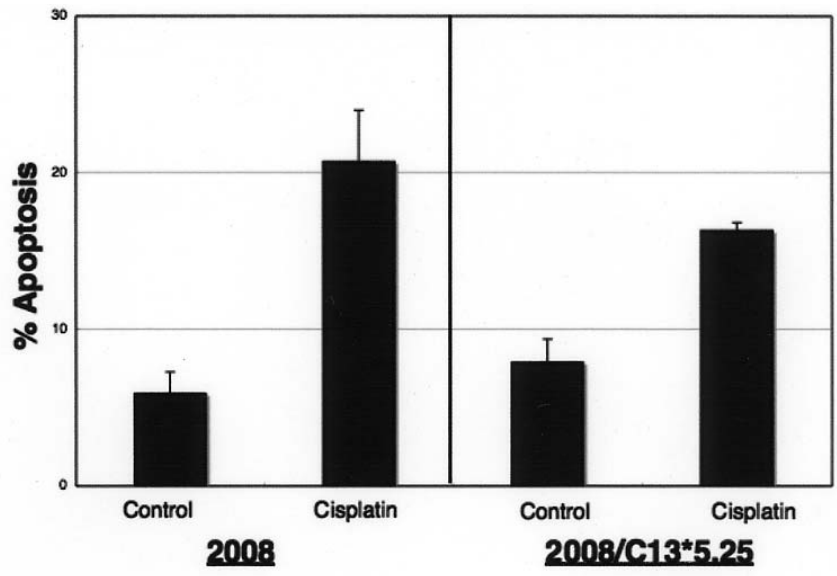

Figure 1. Apoptosis induction by cisplatin in human ovarian carcinoma cells. Cisplatin induced 3.90-fold apoptosis compared to basal apoptotic level in cisplatin-sensitive OV 2008 cells, while it was 2.03-fold in cisplatinresistant $\mathrm{C} 13$ variant cells.

stained cells that contained apoptotic bodies. Fig. 1 shows that, when the cells were evaluated $24 \mathrm{~h}$ after the end of drug exposure, cisplatin induced 3.90 $1.01(\mathrm{SD} ; \mathrm{n}=3)(\mathrm{p}<0.01)$ fold apoptosis compared to basal apoptotic level of $5.04 \pm 1.40(\mathrm{SD} ; \mathrm{n}=3) \%$ to $20.64 \pm 2.34(\mathrm{SD} ; \mathrm{n}=3) \%$ in cisplatin-sensitive OV 2008 cells. Whereas, it induced $2.03 \pm 0.20(\mathrm{SD} ; \mathrm{n}=3)(\mathrm{p}<0.01)$-fold apoptosis compared to basal apoptotic level of $7.83 \pm 1.22(\mathrm{SD} ; \mathrm{n}=3) \%$ to $15.26 \pm 0.54(\mathrm{SD} ; \mathrm{n}=3) \%$ in cisplatin-resistant $\mathrm{C} 13$ cells. This indicats a lower level induction of apoptosis by $50 \%$ in $\mathrm{C} 13$ cells. Thus, we concluded that $\mathrm{C} 13$ cells are resistant to apoptosis.

Inhibitory effect of Ac-DEVD-CHO and caspase-9 inhibitor on cisplatin cytotoxicity. To assess how the caspase pathways involved in the effect of cisplatin, the cells were exposed to cisplatin in the presence or absence of Ac-DEVD-CHO or the caspase-9 inhibitor. The bars in Fig. 2 demonstrate that cisplatin at an $\mathrm{IC}_{50}$ concentration alone produced a $48 \%$ reduction on clonogenic survival in OV 2008 cells and in $\mathrm{C} 13$ cells. Under these culture conditions, Ac-DEVD-CHO blocked cisplatin cytotoxicity and recovered clonogenic survival by $22 \%$ both in OV 2008 and C13 cells. Further, the caspase-9 inhibitor recovered survival by $30 \%$ in OV 2008 and $31 \%$ in $\mathrm{C} 13$ cells. Although these inhibitors slightly decreased their survival, the data suggest that all the caspase cascades are well functioning in resistant cells and that the decreased rate of apoptosis induction might be due to a possible mitochondrial functional change.

Mitochondrial membrane potential ( $\Delta \Psi m)$. To define alterations in mitochondrial function in cisplatin-resistant C13 cells, we assessed the mitochondrial transmembrane potential using potential-sensitive fluorochrome $\mathrm{DiOC}_{6}$. Fig. 3 demonstrates that the polarized cell population in $\mathrm{C} 13$ was 96.5\% determined by the area under the curve in Fig. 3I, while all of the cisplatin-sensitive OV 2008 cells are depolarized (Fig. 3II). This is consistent with the evidence of elevated mitochondrial transmembrane potential in resistant cells.

Detection of mitochondrial DNA damage. Because cisplatin has been reported to cause single strand breaks on incorporation into nuclear DNA after conversion to active metabolites, we investigated whether cisplatin would have a direct damaging effect on mitochondrial DNA. Fig. 4 shows that with a PCR-based method, complete damage to mitochondrial DNA was noted during the treatment of $\mathrm{OV}$ 2008 cells with cisplatin (Fig. 4, lane 5), whereas slight mitochondrial DNA was detectable in C13 cells after incubation with cisplatin (Fig. 4, lane 10). Further, concentration-dependent damage to mitochondrial DNA was detected after incubation with $\mathrm{H}_{2} \mathrm{O}_{2}$ known to induce DNA damage in both cells. After treatment with a maximum concentration of $\mathrm{H}_{2} \mathrm{O}_{2}$, mitochondrial DNA was observed in the $\mathrm{C} 13$ cells whereas complete damage was noted in $\mathrm{OV}$ 2008 cells (Fig. 4, lanes 1-4 and 6-9). This suggests that mitochondria in cisplatin-resistant cells are resistant to cisplatin as well as hydrogen peroxide.

Measurement of oxygen consumption. To monitor the mitochondrial respiratory activity in cisplatin-resistant cells, we focused on the respiration volume as its signal. Respiration

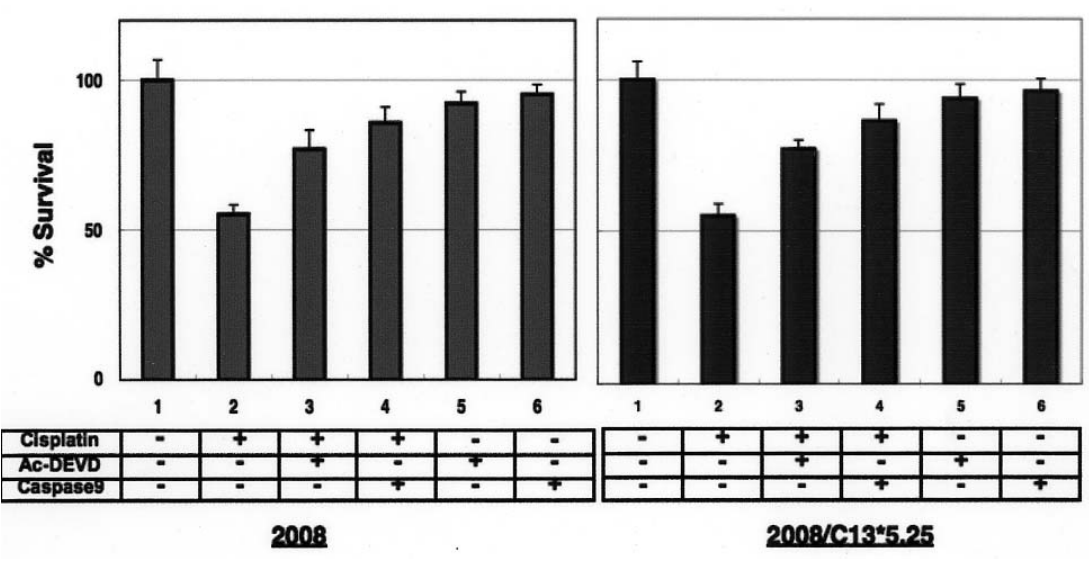

Figure 2. Effect of Ac-DEVD-CHO or the caspase-9 inhibitor on cisplatin cytotoxicity. Ac-DEVD-CHO and the caspase-9 inhibitor blocked cisplatin cytotoxicity and recovered clonogenic survival in OV 2008 and C13 cells to the same degree. 


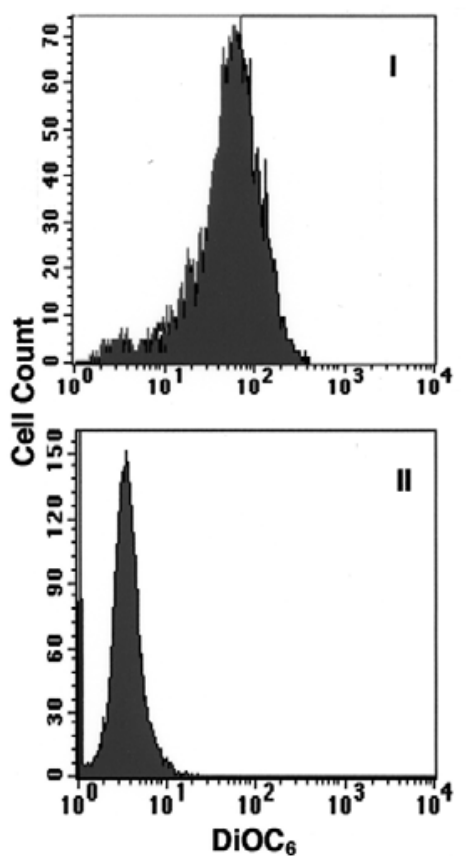

Figure 3. Polarization of mitochondrial membrane in cisplatin resistant cells. Mitochondrial transmembrane potential $\left(\Delta \Psi_{\mathrm{m}}\right)$ was determined using potential-sensitive fluorochrome $\mathrm{DiOC}_{6}$. Polarized cell population in $\mathrm{C} 13$ amount to $96.5 \%$ determined by area under the curve. (I) $\mathrm{C} 13$ cells, (II) OV 2008 cells.

volume was quantified by calculating the continuous oxygen consumption (slop of dissolved oxygen, SDO) and the accumulated total amount of respiration volume (the area under the curve, AUC; subtract AUC of the cells from control AUC). The representative oxygen consumption curves for the cells are shown in Fig. 5 revealing the maximum oxygen consumptions (in SDO's) as $-9,216$ and $-15,629$ for OV 2008 and C13 cells, respectively. The accumulated total amounts of oxygen consumption designated as AUCs are 90,9000 and 67,5000 nano-ampere - min/8 h for OV 2008 and C13 cells, respectively. The data strongly suggest that $\mathrm{C} 13$ cells consumed significantly less oxygen, indicating that these cells were severely deficient in mitochondrial respiration.

\section{Discussion}

Cisplatin is capable of initiating the mitochondrial death pathway directly and inducing tumor cell death $(11,12)$. In the mitochondrial pathway, the instability of mitochondria leads to the redistribution of cytochrome $c$ into the cytoplasm, which initiates the formation of the apoptosome and the sequential activation of caspase- 9 and caspase-3. In this regard, there are three novel observations in this study: i) Despite the significantly decreased apoptotic level in the platinum-resistant cells, the downstream events following the release of cytochrome $c$ from the mitochondria, such as caspase-9, -3, are functioning well in these cells. ii) The mitochondrial DNA in the platinum-resistant cells is highly resistant to cisplatin as well as oxidative stress. iii) Platinumresistant cells decreased oxygen consumption. These results suggest that one of the major cisplatin-resistant mechanisms

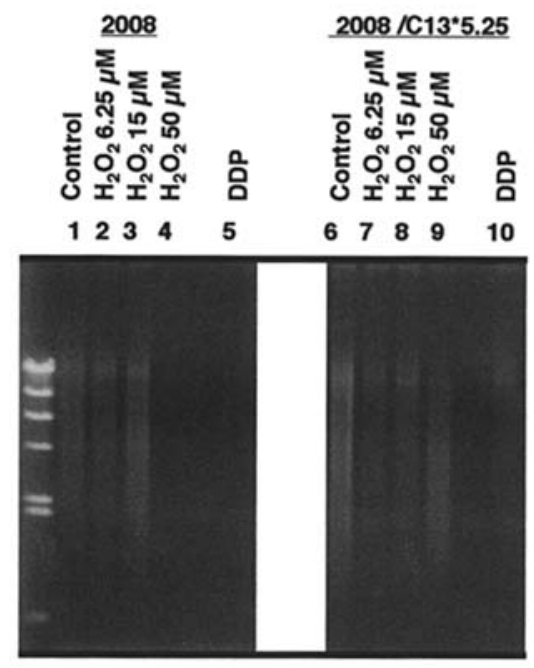

Figure 4. Mitochondrial DNA damage induced by cisplatin and $\mathrm{H}_{2} \mathrm{O}_{2}$ determined by a PCR-based method. Complete damage to mitochondrial DNA was noted during the treatment of OV 2008 cells with cisplatin or $\mathrm{H}_{2} \mathrm{O}_{2}$, while slight mitochondrial DNA was detectable in C13 cells.

lies in the protective function of mitochondria from DNAdamaging agents.

Classical studies have revealed that the drug-resistant phenotype can be significantly associated with resistance to apoptosis $(13,14)$. Our above data strongly indicate that changes at the mitochondrial level are not the secondary characteristics in acquired resistant cells but could impact on the resistance of these cells to undergo drug-induced apoptosis. Alterations in the mitochondrial structure and function occur early during apoptosis before the nuclear or chromatin structure is affected (15). Early findings include a reduction in mitochondrial membrane potential as shown in our data, affected by the opening of permeability transition pores. Regulation of these factors could enhance the platinum sensitivity and reverse the platinum resistance (16).

Cytotoxicity of platinum drugs is based on the interaction with DNA by producing DNA adducts. In eukaryotic cells there are two distinct genomes; one is in the nucleus, and the other is in the mitochondria. These two are able to be targets of platinum attack. Our major interest is how these alterations in mitochondrial DNA characteristics are involved in cisplatin resistance. Park et al argued that the increased manganese superoxide dismutase expression in mitochondrial DNA-depleted SK-Hep1 cells could affect the intracellular metabolism of exogenous reactive oxygen species (7). This indicats that acquired resistance to oxidative stress and doxorubicine in these cells might be the secondary outcome of metabolic change induced by mitochondrial DNA depletion. Whereas, concerning the platinum agents, accumulating evidence has suggested that mitochondrial DNA may be directly involved in the resistance to cisplatin-induced apoptosis. The most important mechanism of cisplatin cell cytotoxicity is platinum-DNA crosslink formation. The study conducted by Liang and Ullyatt (6) implies that mitochondrial DNA-encoded molecules may play a role in the sensitivity of cells to undergo a cisplatin-induced apoptotic death. Our data 


\section{O2 Consumption Curve}

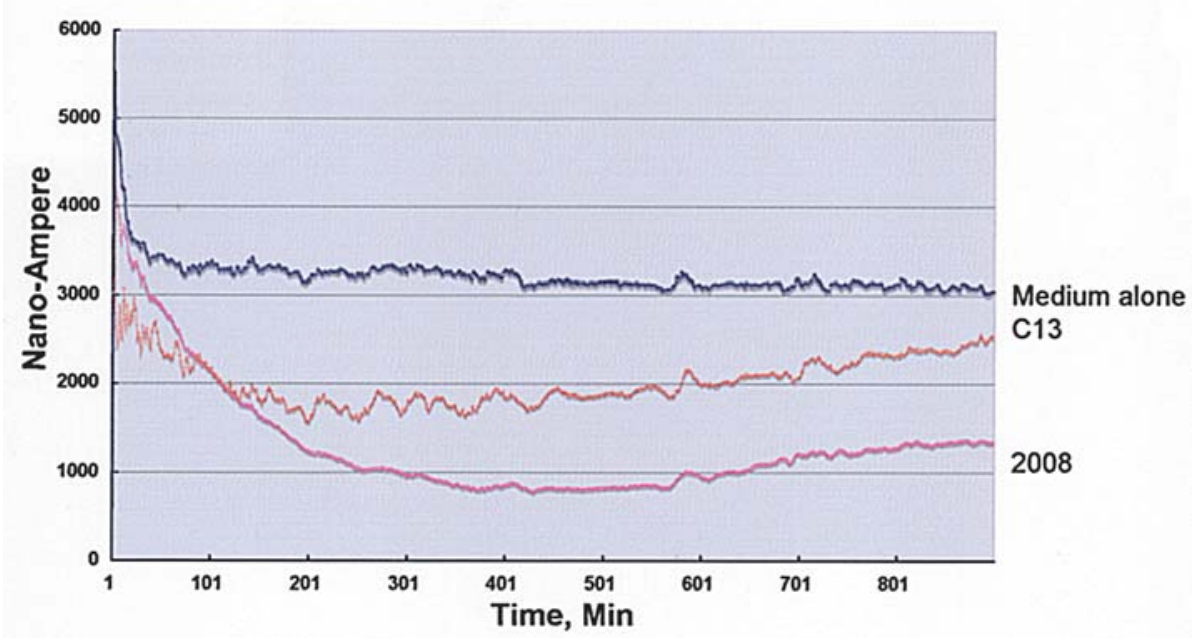

Figure 5. Measurement of oxygen consumption. The respiration volume was quantified by calculating the continuous oxygen consumption (slop of dissolved oxygen, SDO) and the accumulated total amount of respiration volume (the area under the curve, AUC, subtract AUC of the cells from control AUC). C13 cells consumed significantly less oxygen.

demonstrating mitochondrial DNA resistance to cisplatin in platinum-resistant cells provides the possibility that the alteration of mitochondrial DNA resulted in a decreased level of apoptotic induction.

Another point to be addressed is the mechanism of mitochondrial DNA resistance in cisplatin resistant cells. The damage and repair of nuclear DNA has been investigated intensely in cisplatin-resistant cells. Recently, DNA damage and repair has come of interest in that defects in the mitochondrial genomes are associated with a variety of chemotherapeutic agents that work through the mitochondrial DNA damage including platinum $(17,18)$. A higher level and a decreased removal in mitochondrial DNA adducts by cisplatin as compared to nuclear DNA (19) or minimal repair of cisplatin intrastrand crosslinks in mitochondrial DNA (20) have been discussed, however, no correlation between cisplatin resistance and cisplatin-induced mitochondrial DNA damage has been reported. Although our data on platinum resistant cells contain a platinum-resistant mitochondrial DNA,we could not determine whether this was an increased level of removal in mitochondrial DNA adducts or an enhanced repair function of cisplatin intrastrand crosslinks in mitochondrial DNA.

The data presented in this article does not prove that there is an association among the elevated mitochondrial transmembrane potential $(\Delta \Psi \mathrm{m})$, the decreased oxygen consumption, and mitochondrial DNA resistance. No matter how these characteristics might be involved in cisplatin resistance independently or in association with each other, regulating the mitochondrial function could be sufficient to reverse the platinum resistance. Orobol, a phosphatidyl inositol-4 kinase inhibitor, could enhance cisplatin sensitivity in ovarian carcinoma cells by regulating the mitochondrial transmembrane potential $\Delta \Psi \mathrm{m}(16)$. One other method by which one can control the function of mitochondria is to activate the enzyme protein kinase $C$; this endogenous enzyme stimulates the mitochondrial function in the cell (21).
It has been suggested that the activation of cellular protein kinase C could enhance the cell's sensitivity to cisplatin $(22,23)$. Based upon our data, we propose that clinical trials be conducted to test whether the strategies used to control the function of mitochondria may overcome cisplatin resistance.

\section{Acknowledgements}

The authors wish to thank Dr Rakesh Goel, Ottawa Regional Cancer Center, Ottawa, Canada for critical review of this study.

\section{References}

1. Kaufmann SH and Earnshaw WC: Induction of apoptosis by cancer chemotherapy. Exp Cell Res 256: 42-49, 2000.

2. Bratton SB and Cohen GM: Apoptotic death sensor: an organelle's alter ego? Trends Pharmacol Sci 22: 306-315, 2001.

3. Ferri KF and Kroemer G: Organelle-specific initiation of cell death. Nat Cell Biol 3: E255-E263, 2001.

4. Isonishi S, Saitou M, Yasuda M and Tanaka T: Mitochondria in cisplatin resistant cells. Hum Cell 14: 203-210, 2001.

5. Andrews PA and Albright KD: Mitochondrial defects in cisdiamminedichlorocisplatin (II)-resistant human ovarian carcinoma cells. Cancer Res 52: 1895-1901, 1992.

6. Liang $\mathrm{BC}$ and Ullyatt $\mathrm{E}$ : Increased sensitivity to cisdiamminedichloroplatinum induced apoptosis with mitochondrial DNA depletion. Cell Death Differ 5: 694-701, 1998.

7. Park SY, Chang I, Kim JY, Kang SW, Park SH, Singh K and Lee MS: Resistance of mitochondrial DNA-depleted cells against cell death: role of mitochondrial superoxide dismutase. J Biol Chem 27: 7512-7520, 2004.

8. Di Saida PJ, Sinkovics JG, Rutledge FN and Smith JP: Cellmediated immunity to human malignant cells. Am J Obstet Gynecol 114: 979-989, 1972.

9. Reid R, Mar EC, Huang ES and Topal MD: Insertion and extension of acyclic, dideoxy, and ara nucleotides by herpesviridae, human alpha and human beta polymerases: A unique inhibition mechanism for 9-(1,3-dihydroxy-2-propoxymethyl)-guanine triphosphate. J Biol Chem 263: 3898-3904, 1988.

10. Maltese WA and Aprille JR: Relation of mevalonate synthesis to mitochondrial ubiquinone content and respiratory function in cultured neuroblastoma cells. J Biol Chem 260: 11524-11529, 1985. 
11. Dempke W, Voigt W, Grothey A, Hill BT and Schmoll HJ: Cisplatin resistance and oncogenes (Review): Anticancer Drugs 11: 225-236, 2000

12. Melendez-Zajgla J, Cruz E, Maldonado V and Espinoza AM: Mitochondrial changes during the apoptotic process of HeLa cells exposed to cisplatin. Biochem Mol Biol Int 47: 765-771, 1999.

13. Friesen C, Herr I, Krammer PH and Debatin KM: Involvement of the CD95 (APO-1/FAS) receptor/ligand system in druginduced apoptosis in leukemia cells. Nat Med 2: 574-577, 1996.

14. Landowski TH, Gleason-Guzman MC and Dalton WS: Selection for drug resistance results in resistance to Fasmediated apoptosis. Blood 15: 1854-1861, 1997.

15. Kroemer G, Petit P, Zamzami N, Vayssiere JL and Mignotte B: The biochemistry of programmed cell death. FASEB J 9: 1277-1287, 1995.

16. Isonishi S, Saitou M, Yasuda M, Ochiai K and Tanaka T: Enhancement of sensitivity to cisplatin by orobol is associated with increased mitochondrial cytochrome c release in human ovarian carcinoma cells. Gynecol Oncol 90: 413-420, 2003.

17. Singh KK, Russell J, Sigala B, Zhang Y, Williams J and Keslav KF: Mitochondrial DNA determines the cellular response to cancer therapeutic agents. Oncogene 18: 6641-6646, 1999.
18. Pritsos CA, Briggs LA and Gustafson DL: A new cellular target for mitomycin C: a case for mitochondrial DNA. Oncol Res 9: 333-337, 1997.

19. Olivero OA, Chang PK, Lopez-Larraza DM, Semino-Mora MC and Poirier MC: Preferential formation and decreased removal of cisplatin-DNA adducts in Chinese hamster ovary cell mitochondrial DNA as compared to nuclear DNA. Mutat Res 391: 79-86, 1997

20. LeDoux SP, Wilson GL, Beecham EJ, Stevnsner T, Wassermann K and Bohr VA: Repair of mitochondrial DNA after various types of DNA damage in Chinese hamster ovary cells. Carcinogenesis 13: 1967-1973, 1992.

21. Majumder PK, Mishra NC, Sun X, Bharti A, Kharbanda S, Saxena $S$ and Kufe D: Targeting of protein kinase $C$ to mitochondria in the oxidative stress response. Cell Growth Differ 12: 465-470, 2001.

22. Isonishi S, Anrdews PA and Howell SB: Increased sensitivity to cis-diamminedichloroplatium(II) in human ovarian carcinoma cells in response to treatment with 12-O-tetradecanoyl-phorbol13-acetate. J Biol Chem 265: 3623-3627, 1990.

23. Basu A, Teicher BA and Lazo JS: Involvement of protein kinase $\mathrm{C}$ in phorbol ester-induced sensitization of HeLa cells to cisdiamminedichloroplatinum(II). J Biol Chem 265: 8451-8457, 1990. 